\title{
Effects of CMC on Student Participation Patterns in a Foreign Language Learning Environment
}

\author{
James M. Hudson \\ College of Computing \\ Georgia Institute of Technology \\ Atlanta, GA 30332-0280 USA \\ +14048949761 \\ jhudson@cc.gatech.edu
}

\author{
Amy Bruckman \\ College of Computing \\ Georgia Institute of Technology \\ Atlanta, GA 30332-0280 USA \\ $+14048949222$ \\ asb@cc.gatech.edu
}

\begin{abstract}
In this paper, we describe a study examining how communication patterns compare between a traditional foreign language learning classroom and a synchronous, text-based CMC environment. We present suggesting that conversation patterns change significantly when discussions move online. The slight time delay in composing replies and the development of a sense of community seem to be important mechanisms leading to this change.
\end{abstract}

\section{Keywords}

CSCL/Collaborative learning, Education applications, Evaluation, Computer mediated communication

\section{INTRODUCTION}

How does conversational activity change when discussions are mediated by different technologies? Where does synchronous computer-mediated communication (CMC) fall on the continuum between literacy and orality? What are the implications for HCI? In order to begin answering these questions, we examined foreign language learning classrooms engaging in both face-to-face conversations and online conversations. The classroom conversations took place during regularly scheduled class sessions in secondyear French classrooms. The online sessions used IRC Français, an Internet Relay Chat (IRC) client designed specifically for foreign language learning, to engage in discussions outside of class time [1]. We were particularly interested in issues of inhibition since it is frequently cited as an important difficulty for language learning [3]. At the same time, research suggests that more disinhibited behavior often arises on the Internet $[2,4]$. Therefore, we were seeking to understand how the conversational medium affects inhibition in discourse.

\section{STUDY DESIGN}

In this study, we recruited four second-year college French classes to participate in conversations in the classroom and online. We randomly selected two of these classrooms for closer observation. In the classroom, teachers structured their classes as they would without the presence of the researchers. In addition, students met online using IRC Français for one hour each week to participate in synchronous, text-based conversations. From the videotapes of these classes, we intentionally selected typical class sessions for transcription and further analysis.

We also had each of the instructors and a native Frenchspeaking graduate student act as hosts for IRC Françaisbased discussions. Every week, we scheduled three, onehour discussions and required each student to attend one of his/her choosing. The IRC Français software ${ }^{1}$ recorded transcripts of each conversation for analysis. Finally, we conducted in-depth interviews with five students and two professors.

CONVERSATIONS IN THE TRADITIONAL CLASSROOM Conversations in the traditional classroom tend to be marked by patterns of teacher dominance. In the conversations we examined, one teacher, Philippe, spoke approximately $84 \%$ of the total words spoken. A comparison of word count by participant is shown in Figure 1. More interesting, however, is the pattern of discussion that occurred in these conversations. This pattern is shown in Figure 2. When Philippe opened the discussions by asking a general question, he generally faced silence as students attempted to avoid being called upon. Eventually, he called on a student in order to get a response. Philippe and the student, then, engaged in a mini one-on-one conversation before he moved on to another student. In this way, he became the pivotal figure in all conversations; all student comments passed through Philippe. In this paper, we have focused on only one teacher in the interest of space. Other teachers in our study show similar patterns irrespective of class size.

\section{CONVERSATIONS USING IRC FRANÇAIS}

When conversations moved online, a completely different pattern of interaction arose. Upon Philippe asking a question about the chosen topic, nearly every student answered with no prompting. When the students found the chosen topic boring, they said so and offered alternative topics. Students also began addressing one another without the mediation of the instructor.

\footnotetext{
${ }^{1}$ http://www.cc.gatech.edu/elc/irc-francais/
} 


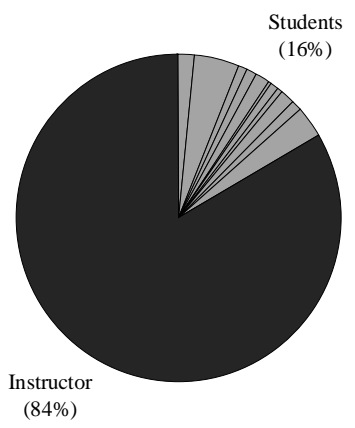

Figure 1: In Philippe's classroom, he speaks a far greater percentage of the words than all students combined.

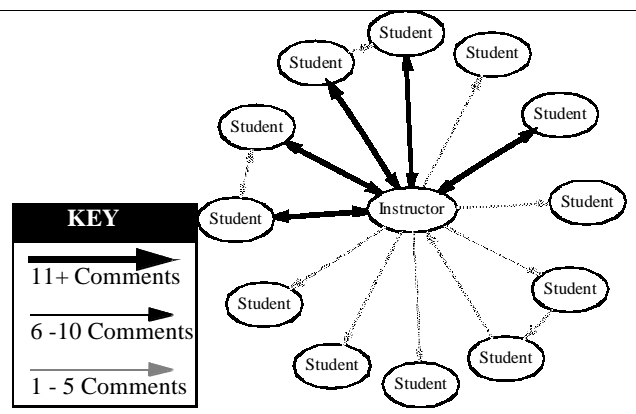

Figure 2: In Philippe's classroom, an analysis of communication patterns shows him as the pivotal figure.

Figure 3 and Figure 4 illustrate data from Philippe's online conversations. During these conversations, his interaction shows a nearly complete reversal. Online, he only spoke approximately $14 \%$ of the total words. A graph of the interaction patterns shows that many more students were talking with one another than happens in the classroom.

\section{POTENTIAL EXPLANATIONS}

In the online conversations, we see different interaction patterns occur among students than in the classroom. Our study pointed to a number of mechanisms leading to this change. First, the fact that messages can be composed before they are posted - message writing is "almost realtime" - allows beneficial thinking time according to the students we interviewed. Also, the sense of community that developed from the online interactions provided important emotional supports for the learning. Many students stated that they developed relationships online that they never before developed in the classroom.

At the same time, new social expectations began to create problems. Relaxed social expectations lead to students approaching the teacher with more informality, but also lead to students arriving late to conversations and leaving early. Future study will focus on understanding and correcting these difficulties.

Future study will further examine how synchronous CMC relates to both orality and literacy. The "typing"

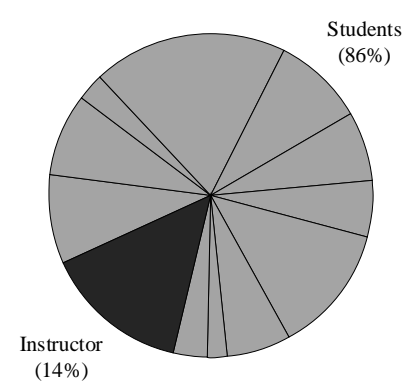

Figure 3: Using IRC Français, Philippe speaks only $14 \%$ of the time, a complete reversal from his behavior in the traditional classroom.

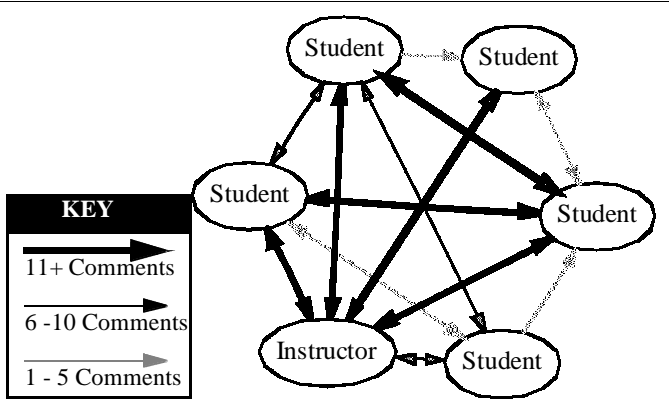

Figure 4: On IRC Français, the communication patterns of Philippe's class becomes much more egalitarian.

interactions on IRC Français occur in a different medium from the oral interactions in a face-to-face classroom. Our next study will begin to examine how these interactions relate to both oral production and written composition.

\section{ACKNOWLEDGMENTS}

We thank PJ Packman for her help in preparing this data. Research in the Electronic Learning Communities (ELC) group (http://www.cc.gatech.edu/elc/) is sponsored by the NSF, IBM, Intel, Microsoft, Neometron, and Ricoh.

\section{REFERENCES}

1. Hudson, J.M. and Bruckman, A. IRC Français: The Creation of an Internet-based SLA Community. Computer Assisted Language Learning.

2. Joinson, A. Causes and Implications of Disinhibited Behavior on the Internet. in Gackenbach, J. ed. Psychology and the Internet: Intrapersonal, Interpersonal, and Transpersonal Implications, Academic Press, San Diego, 1998.

3. Schumann, J.H. Social and Psychological Factors in Second Language Acquisition. in Richards, J.C. ed. Understanding Second and Foreign Language Learning: Issues and Approaches, Newbury House Publishers, Inc., Rowley, 1978.

4. Sproull, L. and Kiesler, S. Connections: New Ways of Working in the Networked Organization. MIT Press, Cambridge, 1991. 